\title{
GENERAL THORACIC SURGERY
}

\section{HUMAN MESOTHELIOMAS CONTAIN THE SIMIAN VIRUS-40 REGULATORY REGION AND LARGE TUMOR ANTIGEN DNA SEQUENCES}

Harvey I. Pass, MD ${ }^{\mathrm{a}, \mathrm{b}}$

Jessica S. Donington, $\mathrm{MD}^{\mathrm{b}}$

Peter $\mathrm{Wu}, \mathrm{MD}^{\mathrm{b}}$

Paola Rizzo, $\mathrm{PhD}^{\mathrm{c}}$

Michael Nishimura, $\mathrm{PhD}^{\mathrm{b}}$

Ronald Kennedy, $\mathrm{PhD}^{\mathrm{d}}$

Michele Carbone MD, $\mathrm{PhD}^{\mathrm{c}}$
Background: A cohort (20\%) of patients with mesothelioma will not have an exposure to asbestos. Recently, a DNA tumor virus (simian virus 40) has been shown to cause hamster mesotheliomas; we previously described simian virus 40-like DNA amino terminus sequences in 29 of 48 mesotheliomas. We analyzed an additional 42 mesotheliomas to determine (1) whether our initial observations were durable and (2) the extent to which the simian virus 40 genome is present in mesotheliomas. Methods: Genomic DNA was extracted from snap frozen mesothelioma tumor samples and from the simian virus 40-induced hamster mesothelioma tumor H9A. Polymerase chain reaction primers were used to amplify various simian virus 40 large $T$-antigen regions including a 105-base pair amino terminus fragment, a 281-base pair carboxyl terminus fragment, and a 310-base pair fragment of the enhancer promoter region. Endonuclease digestions and Southern blotting were used to verify the expected product. Results: Thirty of the $42(71 \%)$ samples amplified T-antigen amino sequences, and specificity was verified by Southern hybridization. Sixteen of 42 samples $(38 \%)$ amplified the appropriate size fragment for the carboxyl terminus, and digestion with BsaB1 matched that of H9A. Twenty-two of 42 samples (52\%) amplified simian virus 40 regulatory sequences and Fok1 digestion matched that of the hamster control tumor. Sequence analysis (4 patients) revealed $100 \%$ homology with the regulatory region of simian virus 40 strain 776. Conclusions: These data suggest an association between the simian virus 40 virus and human mesothelioma that could be exploited for diagnostic/therapeutic options including early detection and potential vaccination strategies. (J Thorac Cardiovasc Surg 1998;116:854-9)
$\mathrm{H}^{-1}$ uman malignant pleural mesothelioma, an asbestosrelated tumor that originates from the serosal lining of the pleura, has no effective therapy and results in a

From the Karmanos Cancer Institute Aerodigestive Program, Detroit,

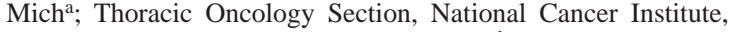
National Institutes of Health, Bethesda, $\mathrm{Md}^{\mathrm{b}}$; Loyola University Cancer Center, Maywood, Illc; and the Department of Microbiology, University of Oklahoma, Oklahoma City, Okla. ${ }^{\mathrm{d}}$

Read at the Seventy-eighth Annual Meeting of The American Association for Thoracic Surgery, Boston, Mass, May 3-6, 1998.

Received for publication May 8, 1998; revisions requested June 11,

1998; revisions received June 23, 1998; accepted for publication June 24, 1998.

Address for reprints: Harper Hospital, 3990 John R, Suite 2102, Detroit, MI 48201.

Copyright $@ 1998$ by Mosby, Inc.

$0022-5223 / 98 \$ 5.00+0 \quad \mathbf{1 2 / 6 / 9 2 8 0 8}$ median survival of 8 to 12 months in the 3000 new cases reported each year in the United States. ${ }^{1}$ At least $20 \%$ of the mesotheliomas in the United States are not associated with asbestos exposure, and less than $5 \%$ of people exposed to a high dose of asbestos experience the development of mesotheliomas. Thus researchers have proposed that additional carcinogens might be responsible for mesotheliomas in non-asbestosexposed individuals or might render particular individuals more susceptible to the carcinogenic effects of asbestos.

Cicala and coworkers ${ }^{2}$ and Sweet and Hilleman ${ }^{3}$ reported that epithelial, spindle cell, and more often mixed-type mesotheliomas, which could be characterized as macroscopically, microscopically, ultramicroscopically, and immunohistochemically identical to 


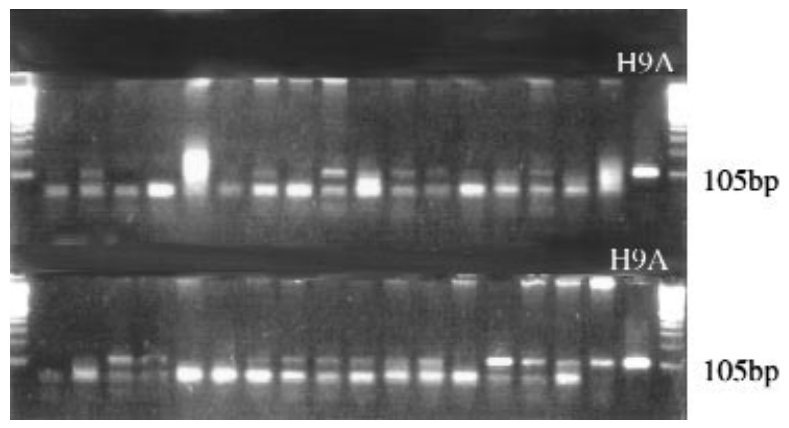

Fig 1. Ethidium bromide gel of PCR products from amplification for Tag amino terminus. Each lane represents a different human mesothelioma, with molecular weight markers on the edges. H9A: amplification product from SV40-induced hamster mesothelioma, positive control.

those seen in humans, developed within a 6-month period in 30 of the 43 hamsters injected with simian virus 40 (SV40), a DNA virus that was a known contaminant of the polio and influenza vaccines in the late 1950s. In collaboration with Carbone and coworkers, ${ }^{4}$ our group previously reported that $60 \%$ (25 of 48 ) of human mesotheliomas contain DNA for the amino terminus region of $\mathrm{T}$ antigen (Tag), a protein associated with various DNA viruses, including SV40, which is capable of causing malignant transformation. Neither the origin nor the true identity of the virus responsible for the Tag to be present in human mesotheliomas could be answered with these preliminary data. Therefore to determine whether the presence of these viral sequences indeed represented previous human infection with SV40, further characterization by amplifying different regions of the SV40 genome, including the regulatory region, and the carboxyl terminus of the Tag was performed. This report summarizes the studies that used the mesothelioma tissue bank generated at the National Cancer Institute from a second series of 42 patients with mesothelioma since the original report. ${ }^{4}$ The purpose of such a study was to (1) verify the findings of SV40-like DNA as previously reported, (2) attempt to characterize other regions of the SV40 genome, and (3) determine whether the amplified regions were consistent with SV40 or another papovavirus.

\section{Methods}

Tissue samples and DNA preparation. From March 1993 to May 1995, portions of 42 consecutive cases of pleural and peritoneal mesotheliomas were snap frozen in the operating room. Patients gave informed consent for use of their tissue in all cases. Genomic DNA was extracted with a commer-

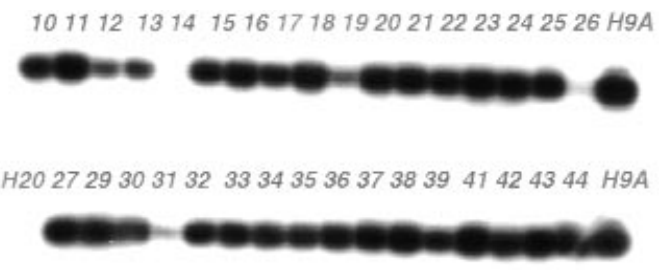

Fig 2. Southern blot hybridization with a specific probe for the RB-binding region of Tag after transfer of the gel seen in Fig. 1. H2O: Lane containing only water, negative control.

cially available kit (Stratagene, La Jolla, Calif) with modifications to ensure for extraction of episomal DNA. These modifications included spinning at $6000 \mathrm{rpm}$ after addition of phenol and no spooling of the DNA after precipitation with ethanol. The DNA was centrifuged at 11,000 rpm in place of spooling. Genomic DNA extracted from the SV40-induced hamster mesothelioma, H9A, was used in all amplifications as a positive control.

Polymerase chain reaction analyses. The suitability of the DNAs was tested with specific primers for the Alu sequences and/or the $\beta$-globin gene (data not shown). The polymerase chain reactions (PCRs) for SV40-sequence amplifications were performed as previously described. ${ }^{4}$ Briefly, hot-start PCR was performed with Amplitaq DNA polymerase (Perkin Elmer Cetus, Norwalk, Conn) according to the vendor's protocol. Thermocycling was performed by denaturation at $94^{\circ} \mathrm{C}$ for 3 minutes, followed by cycling 45 times at $94^{\circ} \mathrm{C}$ for 1 minute, at $55^{\circ}$ to $65^{\circ} \mathrm{C}$ for 1 minute, and at $72^{\circ} \mathrm{C}$ for 1 minute. All amplifications were performed with a positive control (H9A) and negative controls (water only) to guard against possible misinterpretation because of contaminating DNA.

Specific amplification of the SV4O genome

TAG AMINO TERMINUS. PCR primers were used to amplify the RB-pocket binding domain of Tag, which is the region that binds and inactivates the retinoblastoma gene product. Attempted amplification of this site was chosen because the association of Tag with tumor suppressor genes is thought to be important in SV40-mediated transformation. Therefore it was unlikely that this region of Tag would be mutated or deleted if Tag played any role in the development of mesothelioma. Two sets of primer pairs were studied, including the 105 base pair (bp) specific for the RB-pocket binding domain of Tag (Svfor3,Svrev; 5'-TGAGGCTACTGCTGACTCTCAACA-3', 5'-GCATGACTCAAAAAACTTAGCAATTCTG-3') and another set of primers (SVfor2,Svrev; 5'-CTTTGGAGGCTTCTGGGATGCAACT-3', $\mathbf{5}^{\prime}$-GCATGACTCAAAAAACTTAGCAATTCTG-3'), which amplified the RB-pocket binding domain and the intron region of SV40 large Tag (total size, $574 \mathrm{bp}$ ). ${ }^{5}$

SOUTHERN BLOT. PCR products were separated by electrophoresis in 3\% Nusieve/1\% Seakem agarose gels and transferred to a nylon membrane (Hybond N+; Amersham Pharmacia Biotech, Piscataway, NJ). Filters were exposed to 


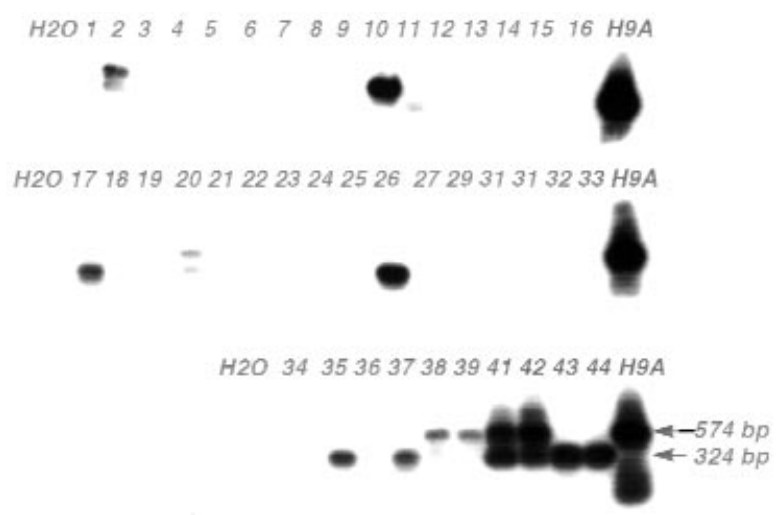

Fig 3. Southern blot hybridization of the PCR products obtained using the primers SVfor2 and SVrev for the amino terminus region of Tag in human mesotheliomas. In addition to the expected 574-bp product, some mesotheliomas contain shorter sequences that are amplified with these SV40-specific primers and that hybridize with an SV40 probe. Sequence analyses indicate that these sequences correspond to Tags with deletions within the intron region.

$1200 \mathrm{~mJ} / \mathrm{cm}^{2}$ of UV light, baked at $80^{\circ} \mathrm{C}$ under vacuum for 2 hours, and hybridized with $3 \times 10^{6} \mathrm{cpm} / \mathrm{mL}$ of a probe representing an internal region of the PCR products. SV-A Probe (5'-GGAAAGTCCTTGGGGTCTTCTACC-3') was used for the amino terminus of Tag amplified using the Svfor3 primers, and SV Probe (5'-ATGTTGAGAGTCAGCABTAGCC-3') was used for the amino terminus of Tag amplified using the SVfor 2 primers. Filters were then washed to a final stringency of $0.5 \times \mathrm{SSC} / 0.1 \% \mathrm{SDS}$ at $52^{\circ} \mathrm{C}$ for $30 \mathrm{~min}$ utes and exposed 1 hour at room temperature.

TAG CARBOXYL TERMINUS. The carboxyl terminus of Tag is highly variable in an evolutionary sense, because this region shows considerable differences among different types of polyomaviruses. This variability could be useful to identify specific viruses and potentially to explain differences in site specific replication. The primer sets for the Tag carboxyl terminus $\quad \mathbf{( 7 , 8 ;} ; \mathbf{5}^{\prime}$-GATGCTATTGCTTTATTT-3', $\mathbf{5}^{\prime}$ GCCAGGAAAATGCTGATA-3') were used to amplify a 281-bp region which contained a unique $B s a B 1$ restriction site. $^{6}$

SV40 REGULATORY REGION. Promoter regions are DNA sequences that form a complex with RNA polymerase and transcription factors to start the process of transcribing DNA to RNA (transcription). Enhancer regions, when present, will subsequently increase the level of transcription of the DNA from a given promoter. It is known that the promoter/ enhancer (ie, regulatory) regions of polyomavirus such as SV40 will influence what site or tissue the virus will replicate. Moreover, the sequence and number of base pairs in the regulatory region could be different among polyomaviruses and could, if detected and sequenced, give insight as to which specific virus was associated with mesothelioma. The primer

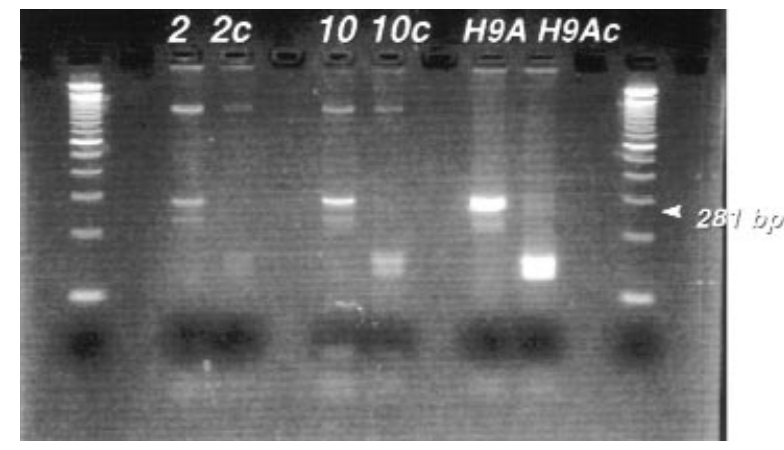

Fig 4. Representative ethidium bromide gel of PCR product of amplification for Tag carboxyl terminus region. Both human mesothelioma specimens 2 and 10 amplify the same product as the control hamster mesothelioma H9A, and after digestion with the restriction endonuclease $B s a B 1$, identical products are formed $(2 \mathrm{c}, 10 \mathrm{c}$, and $\mathrm{H} 9 \mathrm{Ac})$.

pairs R1 and R2 (5'-AATGTGTGTCAGTTAGGGTGTG, TCCAAAAAAGCCTCCTCACTACTT-3') were used to amplify a 315-bp region of the regulatory region for SV40. The region amplified contained a unique Fokl restriction site. $^{7}$

DNA cloning, isolation, and sequencing analysis. PCR products amplified from 4 patients with the R1 and R2 primers were cloned into TA Cloning vector pCRII or PCR2.1 according to the protocol supplied with the original TA Cloning kit (Invitrogen Corporation, Carlsbad, Calif). Recombinant DNA was prepared from ampicillin-resistant transformants ( $E$ coli, INVaF' One Shot; Invitrogen) with the Wizard Plus Minipreps kit (Promega, Madison, Wis). Each DNA was digested with BstXI, electrophoresed on a 7\% polyacrylamide gel, and stained with ethidium bromide to identify those clones containing an SV40 DNA insert. Clones containing SV40 DNA fragments of expected size were sequenced manually by the method of Sanger and coworkers. ${ }^{8}$

\section{Results}

Tag amino terminus. SV40-like DNA was detected in the mesothelioma specimens, confirming our findings in our original report. There was an increased amplification of the 105-bp segment of the amino terminus not only on the ethidium bromide gels ( 30 of $42,71 \%$ ) but also after hybridizing with a specific probe (31 of 33, $92 \%)$. The number of bands originally detected by ethidium bromide with the primers that amplified a larger segment of Tag was much lower. However, Southern blot demonstrated a $25 \%$ amplification rate for the larger region of amino terminus Tag with the $\mathrm{SVfor} 2$ primers. Two species were identified in some of the specimens representing amplification of the intact segment (no intron deletion, 574 bp) or with an intron deletion (324 bp). 


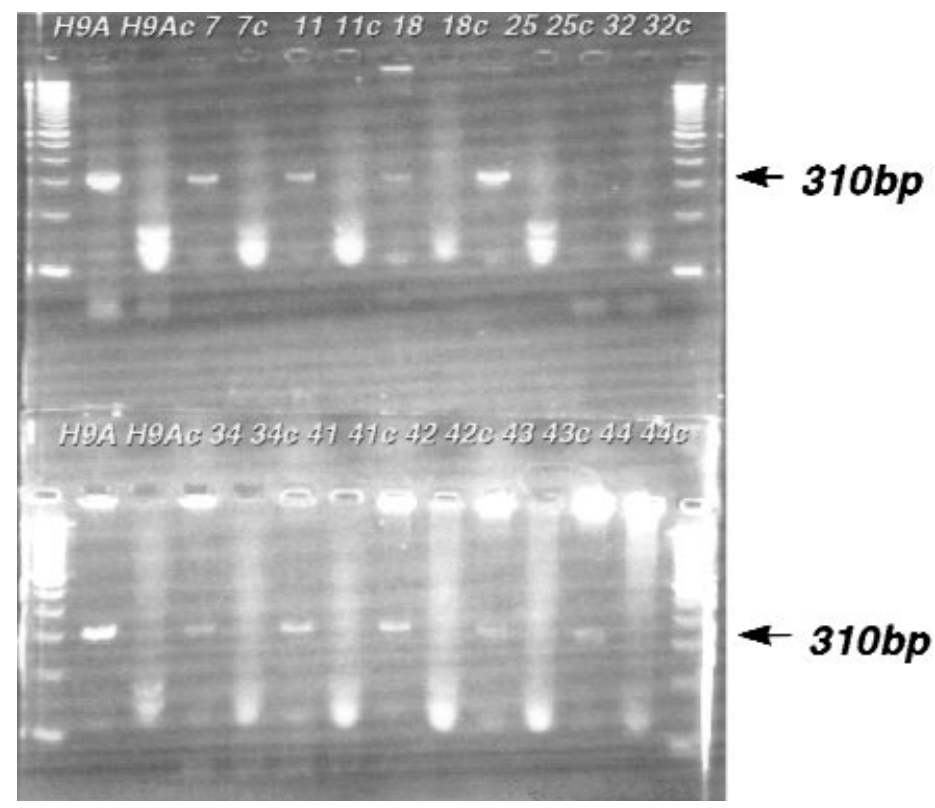

Fig 5. Representative ethidium bromide gel of PCR product of amplification for SV40 regulatory region. Each lane represents a different human mesothelioma, with molecular weight markers on the edges. A 310-bp product of the regulatory region is amplified by the human mesotheliomas and is cut identically as the control hamster mesothelioma H9A, with the restriction endonuclease Fok 1 .

Tag carboxyl terminus. Sixteen of the 42 specimens (38\%) contained sequences for the carboxyl terminus of Tag. The digestion patterns after $B s a B l$ were identical to that seen with $B s a B l$ digestion of the product obtained after carboxyl terminus amplification of the H9A hamster mesothelioma.

Regulatory sequences. Fifty-two percent (22 of 42 specimens) had amplification of the regulatory region seen on ethidium bromide gels. The digestion patterns after Fokl were identical to that seen with Fokl digestion of the hamster mesothelioma control.

Sequencing of the amplification product of the regulatory region in 4 patients revealed an identical sequence to that seen with the hamster mesothelioma, which, in fact, was derived from wild type strain SV40 776.

Overall, 24\% of the mesothelioma studies had amplification of all 3 regions (ie, amino and carboxyl terminus for Tag as well as the regulatory region for SV40).

\section{Discussion}

This is the second report from our laboratory that details the presence of viral DNA in human mesothelioma. When we began this second set of investigations, there were no other reports in the literature that verified these data, and because SV40 was not believed to be a human pathogen, skepticism for a viral connection to this tumor was significant. The findings were thought to be the result of "PCR contamination" despite the fact that great efforts to avoid contamination by extracting the DNA from 1 SV40 free lab and by running the PCR reactions and Southern blot experiments in another building.

We felt that repeating the amplifications reported in the first series in a second set of patients could not only further convince us and others of the authenticity of these findings but also would allow us to further identify other regions of the supposed viral genome if they were present. Such an approach would narrow the field of possible mesothelioma-associated viral candidates.

Since the publication of our original report, the National Institutes of Health have sponsored a workshop specifically devoted to the issue of whether SV40 is a human pathogen and there have been confirmatory data, specifically in patients with mesothelioma, that sequences for the amino terminus of Tag are present. ${ }^{9}$ Unfortunately, however, there have been no data that shed light on the origins of the mesothelioma-associated virus or the strain of the associated virus. The theories with regard to the origins of the virus, however, have been the subject of a new series of controversial epidemiologic and genetic studies caused by the known 
contamination of the original poliovaccines and adenovaccines with SV40. ${ }^{10-12}$

From the data in this report, it appears as if the fingerprint of SV40 itself is becoming more prominent as the polyomavirus associated with mesothelioma. Not only have we confirmed that the RB-pocket binding region of the amino terminus of Tag is present in our specimens, but we have also reported that the incidence is even higher if one carefully extracts the DNA to make sure one is not only harvesting DNA potentially integrated in the genome but also existing episomally. The RB-pocket binding region is a highly conserved area because it plays a critical role in the process of SV40 Tag-mediated transformation. Therefore mutations should not be expected in this region if a Tag-like protein contributes to tumor development. On the other hand, the intron region could likely contain deletions and/or mutations, which has been confirmed in osteosarcomas that express Tag. This could, among other reasons, explain why the percentage of positive amplification results were different in this study between the primers that both amplify the RB-binding region.

The carboxyl end of Tag is highly variable in an evolutionary sense, because this region shows considerable divergence in different papovaviruses. The primers used in this study to amplify the carboxyl terminus were specific for SV40 strain 776 , further strengthening the suggestion that the associated virus is SV40. There are sequences at the carboxy terminus of Tag that will determine the host range in cultured cells; these sequences are different among different histologic organ types infected with SV40 (ie, kidney versus brain). These data suggest that sequence differences at the carboxy end of Tag may contribute to tissue-specific SV40 replication. How this occurs is unknown, specifically as to why mesothelium would be targeted, but Tag likely needs to interact with host cell transcription factors in the process of switching from early to late transcription, and transcription factors vary in a cell type and tissue-specific fashion.

Further identification of the strain of virus could come from characterization of the regulatory region. It is significant in itself that regulatory regions compatible with SV40 are present in 52\% of the specimens, and sequencing from 4 patients reveals that they have base pair homology with wild type 776 SV40.

This study does not give insight into the origin of the sequences, nor does it implicate SV40 as a complete carcinogen in mesothelioma. Morever, as described in our previous study, most of these patients have asbestos fibers in their lungs. These data do substantiate that SV40 sequences are consistently present in mesothe- lioma. Recent data, however, suggesting that natural isolates of SV40 that originate in rhesus monkeys can exist in multiple forms and that maternal-infant transmission of the virus may occur heightens awareness for this polyomavirus as a potential human pathogen. ${ }^{13,14}$

\section{REFERENCES}

1. Pass HI, Pogrebniak HW. Malignant pleural mesothelioma. Curr Probl Surg 1993;30:921-1020.

2. Cicala C, Pompetti F, Carbone M. SV40 induces mesotheliomas in hamsters. Am J Pathol 1993;142:1524-33.

3. Sweet BH, Hilleman RM. The vacuolating virus SV40. Proc Soc Exp Biol Med 1960;105:420-7.

4. Carbone M, Pass HI, Rizzo P, et al. Simian virus 40-like DNA sequences in human pleural mesothelioma. Oncogene 1994;9: 1781-90.

5. Carbone M, Rizzo P, Procopio A, et al. SV40-like sequences in human bone tumors. Oncogene 1996;13:527-35.

6. Ilyinskii PO, Muthiah DD, Horvath CJ, Desrosiers RC. Genetic analysis of simian virus 40 from brains and kidneys of Macaque monkeys. J Virol 1992;66:6353-60.

7. Lednicky JA, Garcea RL, Bergsagel DL, Butel JS. Natural simian virus 40 strains are present in human choroid plexus and ependymoma tumors. Virology 1997;212:710-7.

8. Sanger F, Fritsch EF, Coulson AR. DNA sequencing with chainterminating inhibitors. Proc Natl Acad Sci 1977;73:5463-7.

9. Carbone M, Rizzo P, Pass HI. Simian virus 40, poliovaccines and human tumors: a review of recent developments. Oncogene 1997; 15:1877-88.

10. Strickler HD, Goedert JJ, Felming M, et al. Simian virus 40 and pleural mesothelioma in humans. Cancer Epidemiology, Biostatistics, and Prevention 1995;5:473-5.

11. Strickler HD, Rosenberg PS, Devesa SS, Hertel J, Fraumeni JF $\mathrm{Jr}$, Goedert JJ. Contamination of poliovirus vaccines with simian virus 40 (1955-1963) and subsequent cancer rates. JAMA 1998;279:292-5.

12. Shah KV, Daniel RW, Strickler HD, Goedert JJ. Investigation of human urine for genomic sequences of the primate polyomaviruses simian virus 40 , BK virus and JC virus. J Infect Dis 1997; 176:1618-21.

13. Lednicky JA, Arrington AS, Stewart AR, et al. Natural isolates of simian virus 40 from immunocompromised monkeys display extensive genetic heterogeneity: new implications for polyomavirus disease. J Virol (In press).

14. Jafar S, Rodriquez-Barradas M, Graham DY, Butel JS. Serological evidence of sv40 infections in HIV-infected and HIVnegative adults. J Mol Virol (In press).

\section{Discussion}

Dr Larry R. Kaiser (Philadelphia, Pa). Can you give us some speculation as to whether you think that SV40 might be involved in the pathogenesis of the disease? And we certainly know that there is a percentage of patients who have no asbestos exposure and yet mesotheliomas develop. We know, for instance, that there is the occasional patient who has been cured of Wilms tumor in whom mesothelioma has developed. We know that WT1 is expressed in mesotheliomas. How would you fit SV40 into the picture? How could there possi- 
bly be a link with asbestos? Maybe you could just postulate on that for us.

Dr Pass. This is very controversial, both economically and public healthwise. $\mathrm{T}$ antigen will combine with retinoblastoma gene product, as well as with the p53 protein product; with these unactivated, you can potentially have transformation.

Asbestos is a known fiber that causes not only chromosomal problems within the human genome but also causes immunosuppressive phenomena. In a situation where you could have chromosomal breaks with oncogene amplification or tumor suppressor gene loss, combined with a virus that would work in an immunosuppressed setting and combined with other tumor suppressor genes, the field may be possibly set for mesothelial carcinogenesis.

There are no data, however, that have demonstrated that giving SV40 virus to human mesothelial cells, either with or without asbestos, can transform cells into mesothelioma.

Dr Kaiser. Where are you heading with this now? I think that it is intriguing both from a diagnostic and a therapeutic possibility. I know in our work we have looked at basically trying to say that T antigen of SV40 may be specific for mesothelioma and perhaps if we are generating an immune response against mesothelioma with some of the adenoviral work, that we should see a response against SV40 T antigen. So that is 1 potential strategy, and interestingly we have seen that. Where are you going with this now?

Dr Pass. Our goal is the usual adoptive immunotherapy approach: to identify specific epitopes for T cells that may be expressed by $\mathrm{T}$ antigen, so that we can then take advantage and use it with peptides to either amplify a patient's own immune system or amplify ex vivo lymphocytes that can be supercharged to hone in on areas specifically that have $\mathrm{T}$ antigen.

Dr David J. Sugarbaker (Boston, Mass). There is a study that is going forward (from the NIH by Howard Strickler and a group in the viral division) that is based on the concept that SV40 is associated with a variety of different tumors (ie, head and neck tumors and other tumors) and that this association with mesothelioma may be more or less a background pickup, a contaminant. So the idea is to move forward with a care- fully constructed set of what they call clean laboratories to be sure there is not this background contaminant.

Could you comment on that concept, particularly in light of your hamster work, and give us an idea as to what you think about it.

Dr Pass. I am aware of that PCR working group because essentially we were part of it in the beginning. The purpose of the group is essentially not only to reconfirm our data but also to set the PCR conditions, because there were questions raised about how many amplifications you have to do.

With regard to other tumors, it should not be propagated that this is a common phenomenon. Our group has published whether SV40 is present in other tumors; the other tumors it is present in include childhood osteosarcomas, of which $40 \%$ of them are present, and ependymomas and choroid plexus tumors. This is not a phenomenon of other tumors.

Dr Adelheid End (Vienna, Austria). Maybe I missed that point in your lecture. Was there a difference between the asbestos and the nonasbestos group concerning the incidence of SV40?

Dr Pass. Essentially if you look at these patients, and we have done this in the first series, $95 \%$ of these patients will have asbestos. We have a cohort of patients within this study in whom indeed we have done fiber analysis who are low asbestos and SV40 positive. We have not analyzed that specifically. But at this point we cannot say that SV40 independently, without a background of asbestos, does anything.

Dr Peter Goldstraw (London, England). Could you speculate as to why different fragments of the virus are persisting in different tumors?

Dr Pass. I think that there is a possibility that there may be a hybrid virus that is actually going on here that we do not know truly. We may have a regulatory region that is completely the same as wild type SV40, but the amino terminus and the carboxy terminus may be different because of mutations; therefore you do not amplify it all the time. This may also explain why you get certain tissue-specific characters. Why do ependymomas or osteosarcomas develop in children, while older individuals seem to have a propensity for having this in mesotheliomas? These mutations may actually dictate where the tumor may have its relevance. 